\title{
Minimizing and Communicating Radiation Risk in Pediatric Nuclear Medicine*
}

\author{
Frederic H. Fahey ${ }^{1,2}$, S. Ted Treves ${ }^{1,2}$, and S. James Adelstein ${ }^{2}$ \\ ${ }^{1}$ Division of Nuclear Medicine and Molecular Imaging, Children's Hospital Boston, Boston, Massachusetts; and ${ }^{2}$ Department of \\ Radiology, Harvard Medical School, Boston, Massachusetts
}

The value of pediatric nuclear medicine is well established. Pediatric patients are referred to nuclear medicine from nearly all pediatric specialties including urology, oncology, cardiology, gastroenterology, and orthopedics. Radiation exposure is associated with a potential, small, risk of inducing cancer in the patient later in life and is higher in younger patients. Recently, there has been enhanced interest in exposure to radiation from medical imaging. Thus, it is incumbent on practitioners of pediatric nuclear medicine to have an understanding of dosimetry and radiation risk to communicate effectively with their patients and their families. This article reviews radiation dosimetry for radiopharmaceuticals and also CT given the recent proliferation of PET/CT and SPECT/CT. It also describes the scientific basis for radiation risk estimation in the context of pediatric nuclear medicine. Approaches for effective communication of risk to patients' families are discussed. Lastly, radiation dose reduction in pediatric nuclear medicine is explicated.

Key Words: pediatrics; radiobiology; dosimetry; radiation risk

J Nucl Med Technol 2012; 40:13-24

DOI: 10.2967/jnumed.109.069609

$\mathbf{P}$ ediatric nuclear medicine provides important and often essential information that assists in the diagnosis, staging, treatment, and follow-up of a variety of disorders including those of the central nervous, endocrine, cardiopulmonary, renal, and gastrointestinal systems, as well as in the fields of oncology, orthopedics, organ transplantation, and surgery. Because of its high sensitivity, nuclear medicine often can detect disease in its earliest stages, allowing for early treatment. The noninvasive nature of nuclear medicine makes it an extremely useful diagnostic tool for the evalu-

\footnotetext{
Received Feb. 15, 2011; revision accepted May 16, 2011.

For correspondence or reprints contact: Frederic H. Fahey, Division of Nuclear Medicine and Molecular Imaging, Department of Radiology, Children's Hospital Boston, 300 Longwood Ave., Boston, MA 02115.

E-mail: frederic.fahey@childrens.harvard.edu

*NOTE: FOR CE CREDIT, YOU CAN ACCESS THIS ACTIVITY THROUGH THE SNM WEB SITE (http://www.snm.org/ce_online) THROUGH MARCH 2012. PARTICIPANTS WHO HAVE ALREADY TAKEN THE EXAM USING JNM AND PASSED CANNOT RETAKE THE EXAM.

COPYRIGHT @ 2012 by the Society of Nuclear Medicine, Inc.
}

ation of children. It provides valuable diagnostic information that may not be easily obtained by other diagnostic imaging methods, some of which may be more invasive or result in higher radiation exposures $(1,2)$.

Nuclear medicine involves the administration of small amounts of radiopharmaceuticals that emit radiations such as $\gamma$-rays, $x$-rays, $\beta$-particles, or positrons. This emission exposes the patient to low levels of ionizing radiation that might lead to detrimental health effects, of which carcinogenesis is the primary concern. In the dose range associated with most nuclear medicine procedures, there are limited human epidemiologic data, and mechanistic biologic observations can be contradictory. Nevertheless, the consensus is that it is prudent to assume that the risk at these lower doses can best be estimated by a linear extrapolation from higher doses for radiation protection purposes (3).

The past quarter-century has seen a remarkable increase in the use of medical imaging (4-6). In the United States, the number of CT scans performed has increased nearly 4-fold from 18 to over 60 million scans a year in the past $25 \mathrm{y}$. The total number of nuclear medicine procedures in the United States has increased by almost 3-fold from 6.3 to 18.1 million procedures from 1984 to 2006 (Fig. 1), with approximately $1 \%$ of these procedures being performed on children (4). When the number and distribution of pediatric radiologic and nuclear medicine procedures in the United States were investigated through a review of health insurance records of over 350,000 children (7), $42.5 \%$ of the children had at least 1 radiologic examination in the $3 \mathrm{y}$ covered by the study. It was estimated that, on average, a child will receive 7 radiologic examinations by the age of 18 . The most common procedure was a plain radiograph ( $84.7 \%$ of the studies performed), followed by CT (11.9\%), fluoroscopy or angiography $(2.5 \%)$, and nuclear medicine $(0.9 \%)$.

The mean annual per capita effective dose from medical radiation in the United States rose from 0.5 to $3 \mathrm{mSv}$ between 1980 and 2006 (Fig. 2) (6). Approximately half of this exposure is due to CT $(1.47 \mathrm{mSv})$, and one quarter is due to nuclear medicine $(0.77 \mathrm{mSv})$. These statistics primarily reflect medical imaging of adults. Most of the cumulative dose for nuclear medicine comes from myocardial 


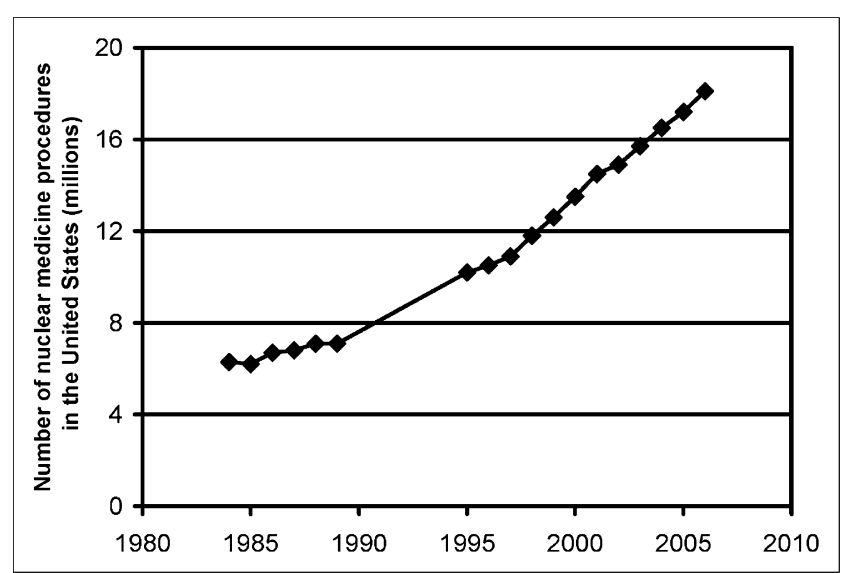

FIGURE 1. Number of nuclear medicine procedures in United States from 1984 to 2006. In this time span, number of nuclear medicine procedures increased by almost factor of 3 , from 6.3 to 18.1 million (4).

perfusion imaging, a procedure not frequently performed on children, with only about $0.1 \%$ of nuclear cardiac scans being performed on children (4). Another study based on health claims of patients 18-65 y old in the United States over a 3-y period estimated a slightly lower mean annual per capita dose from medical radiation $(2.4 \mathrm{mSv})$, perhaps because patients over $65 \mathrm{y}$ and those in the last few months of life were excluded (8). The individual dose distribution is skewed, with a median dose of $0.1 \mathrm{mSv}$. Thirty-one percent of the patients did not receive a radiographic examination during the 3-y study, whereas $80 \%$ received an annual cumulative effective dose from medical imaging of less than $3 \mathrm{mSv}, 18 \%$ received between 3 and $18 \mathrm{mSv}$, and less than $2 \%$ received more than $20 \mathrm{mSv}$. Although these data are in adults, the pediatric population probably shows a similar trend, with most children receiving little or no radiation whereas a few receive a higher cumulative effective dose.

In the United States, there has been recent concern by the media and the public about this increasing use of medical imaging $(9,10)$. The importance of properly trained personnel and adequate quality control programs has been stressed

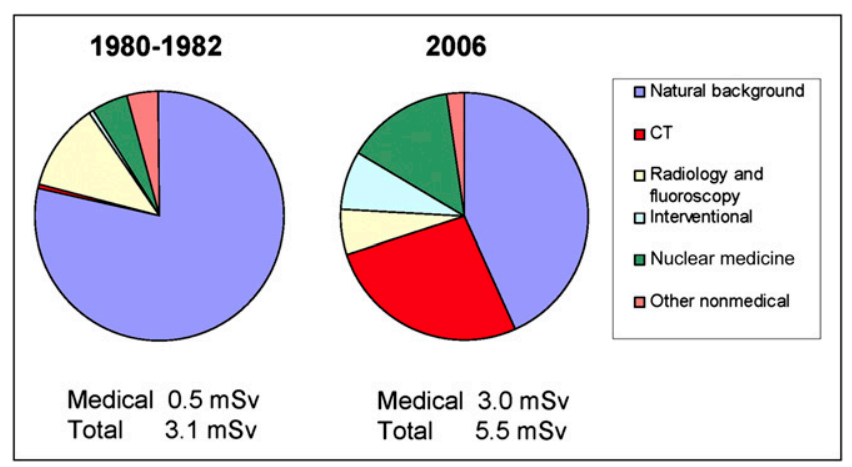

FIGURE 2. Estimated annual per capita adult effective dose in United States. Chart on left illustrates distribution of effective dose in 1980-1982. Chart on right shows distribution in 2006 (6). as a means of protecting the public from exposure to undue levels of radiation $(11,12)$. However, the deliberations of both the public and regulatory agencies have generally not focused on the exposure of children.

To understand the potential hazard resulting from pediatric nuclear medicine, it is essential to have a fundamental understanding of radiation dosimetry, radiation risk, and how this risk may vary in children relative to adults. It is also important to communicate the nature of these risks to our patients and their families. This article will review the basics of radiation dosimetry for nuclear medicine, including hybrid imaging (PET/CT and SPECT/CT). The scientific basis of the estimation of radiation risk will be discussed, with attention to how risk varies with age at the time of exposure. Radiation risk will be discussed in the context of pediatric nuclear medicine so that nuclear medicine practitioners can make informed decisions about its use and are able to discuss radiation risk with their patients and their parents. Lastly, approaches to reducing radiation dose, and thereby risk, from pediatric examinations will be presented.

\section{DOSIMETRY OF PEDIATRIC NUCLEAR MEDICINE}

\section{Dosimetry of Radiopharmaceuticals}

The dosimetry of nuclear medicine can be summarized by the basic equation developed by the MIRD Committee $(13,14)$.

$$
\mathrm{D}\left(\mathrm{r}_{\mathrm{T}}\right)=\sum_{\mathrm{S}} \tilde{\mathrm{A}}\left(\mathrm{r}_{\mathrm{S}}\right) \mathrm{S}\left(\mathrm{r}_{\mathrm{T}} \leftarrow \mathrm{r}_{\mathrm{S}}\right), \quad \text { Eq. } 1
$$

where $\mathrm{D}\left(\mathrm{r}_{\mathrm{T}}\right)$ is the radiation dose to a particular target organ $\left(\mathrm{r}_{\mathrm{T}}\right), \tilde{\mathrm{A}}\left(\mathrm{r}_{\mathrm{S}}\right)$ is the time-integrated activity in a selected source organ $\left(r_{S}\right)$, and $S\left(r_{T} \leftarrow r_{S}\right)$ is the radionuclide-specific quantity representing the mean dose to the target organ per unit activity present in the source organ. $\Sigma_{S}$ indicates summing over all source organs in which the radiopharmaceutical distributes. $\mathrm{S}\left(\mathrm{r}_{\mathrm{T}} \leftarrow \mathrm{r}_{\mathrm{S}}\right)$ is given by

$$
\mathrm{S}\left(\mathrm{r}_{\mathrm{T}} \leftarrow \mathrm{r}_{\mathrm{S}}\right)=\sum_{\mathrm{i}} \Delta_{\mathrm{i}} \phi_{\mathrm{i}} / \mathrm{M}_{\mathrm{T}},
$$

where $\Delta_{\mathrm{i}}$ is the mean energy per nuclear transformation for the $\mathrm{i}^{\text {th }}$ radiation emitted by the radiopharmaceutical, $\varphi_{\mathrm{i}}$ is the fraction of energy emitted by the $i^{\text {th }}$ radiation from the source organ that is absorbed by the target organ, and $\mathrm{M}_{\mathrm{T}}$ is the mass of the target organ. $\Sigma_{\mathrm{i}}$ indicates summing over all radiations, i, emitted from the radiopharmaceutical. For any particular radiopharmaceutical, there may be several source organs that must be considered. For example, with ${ }^{18} \mathrm{~F}$-FDG the source organs include the brain, heart, and liver. For each source organ, the radiation dose is calculated and summed to determine the total dose to the target organ.

The radiopharmaceutical dose to children varies from that to an adult for several reasons, particularly the patient size. The absorbed fractions and organ masses of children 
differ from those of adults because the organs of children are smaller and closer together. Tables of $\mathrm{S}$ values for patients of different ages can be used to estimate the radiation dose to children $(15,16)$. These models traditionally have used simple shapes representing the organs. Voxel-based models are more realistic but more complicated, leading to intensive calculations that can provide more accurate dose estimations (17-19).

Using this method, one can estimate the radiation dose to organs of patients of different sizes and ages. The organ receiving the highest dose is referred to as the critical organ. In addition, one can calculate the effective dose, which is a weighted sum of the individual organ doses based on the biologic radiosensitivity of each organ $(20,21)$. In an adult, the weights are selected such that an effective dose in millisieverts carries the same risk of adverse biologic effect as a uniform whole-body-dose equivalent of the same magnitude. These weights represent crude averages across the adult population (without attention to age and sex) and do not necessarily reflect the risks to the pediatric population.

From these models and assumptions, tables of organ doses and effective doses have been generated for application to the pediatric population (22-26). Table 1 lists dosimetric estimates for several procedures common to pediatric nuclear medicine. It includes estimates of both critical organ and effective doses. For each procedure, a particular maximum administered activity is assumed and the administered activity for smaller patients is scaled by patient weight.
These estimates are averages over a wide range of patients at each age. They do not take into consideration individual differences in anatomy and physiology from the standard models. A particular patient's body may vary from the standard with respect to size, weight, shape, organ orientation, and distances from other organs. Thus, the absorbed fraction and organ mass vary from patient to patient. These models also make assumptions with respect to the amount of radioactivity that went to each source organ, including rates for uptake and clearance of the radiopharmaceutical from that organ. For example, the models for ${ }^{99 \mathrm{~m}} \mathrm{Tc}$-mercaptoacetyltriglycine (MAG3) assume normal clearance from the kidneys, but clearance might not be normal in some patients having this scan. Application of pediatric models is problematic because children can vary greatly in body size and habitus (e.g., one 10-yold may weigh $30 \mathrm{~kg}$ and another $60 \mathrm{~kg}$ ). Also, these models are based on adult physiology, and such a basis may not be appropriate for children. Therefore, the radiation dose to a particular patient may vary by as much as 100\%-200\% from these estimates. These methods were developed for estimating the average dose to a population and should not be used to estimate the dose to a specific patient.

These estimates indicate that the effective dose for typical nuclear medicine procedures is in the same range as many radiographic procedures. Table 2 lists the estimated effective dose to adults for a wide range of imaging procedures, including nuclear medicine (23-26). The estimates for nuclear medicine were taken from the adult effective doses listed in

TABLE 1

Estimates of Critical Organ and Effective Dose for Common Pediatric Nuclear Medicine Procedures

\begin{tabular}{|c|c|c|c|c|c|c|}
\hline & Max admin act (MBq) & $1-y$-old & 5 -y-old & 10-y-old & 15-y-old & Adult \\
\hline Mass (kg) & & 9.7 & 19.8 & 33.2 & 56.8 & 70 \\
\hline 99mTc-MDP* & 740 & & & & & \\
\hline Bone surface (mGy) & & 54.5 & 46.0 & 45.6 & 49.2 & 46.6 \\
\hline Effective dose (mSv) & & 2.8 & 2.9 & 3.9 & 4.2 & 4.2 \\
\hline${ }^{99 \mathrm{mT}} \mathrm{T}-\mathrm{ECD}^{\dagger}$ & 740 & & & & & \\
\hline Bladder wall (mGy) & & 13.4 & 23.0 & 30.5 & 37.2 & 37.0 \\
\hline Effective dose (mSv) & & 4.1 & 4.6 & 5.3 & 5.9 & 5.7 \\
\hline 99mTc-sestamibi & 740 & & & & & \\
\hline Gallbladder (mGy) & & 32.9 & 20.9 & 20.4 & 27.0 & 28.9 \\
\hline Effective dose (mSv) & & 5.4 & 5.9 & 6.3 & 7.2 & 6.7 \\
\hline 99mTc-MAG3* & 370 & & & & & \\
\hline Bladder wall (mGy) & & 17.2 & 19.8 & 31.3 & 44.1 & 42.7 \\
\hline Effective dose (mSv) & & 1.2 & 1.3 & 2.2 & 2.8 & 2.7 \\
\hline $123 \mathrm{I}-\mathrm{MIBG}^{*}$ & 370 & & & & & \\
\hline Liver (mGy) & & 16.6 & 18.5 & 22.4 & 25.6 & 24.8 \\
\hline Effective dose (mSv) & & 3.4 & 3.8 & 4.5 & 5.0 & 4.8 \\
\hline${ }^{18} \mathrm{~F}_{-} \mathrm{FDG}^{\dagger}$ & 370 & & & & & \\
\hline Bladder wall (mGy) & & 25.6 & 35.9 & 44.4 & 48.8 & 50.5 \\
\hline Effective dose (mSv) & & 5.2 & 5.9 & 6.6 & 7.3 & 7.4 \\
\hline
\end{tabular}

*Based on ICRP 80 (25).

${ }^{\dagger}$ Based on ICRP 106 (26).

Max admin act $=$ maximum administered activity is that administered to adult or large child $(70 \mathrm{~kg})$ (administered activities for smaller children are scaled by body weight); ECD = ethylcysteinate dimer; MIBG = metaiodobenzylguanidine. 
TABLE 2

Adult Effective Doses (mSv) for Radiographic and Nuclear Medicine Procedures

\begin{tabular}{lc}
\hline \multicolumn{1}{c}{ Procedure } & $\begin{array}{c}\text { Average effective } \\
\text { dose (mSv) }\end{array}$ \\
\hline $\begin{array}{l}\text { Posterior/anterior and lateral chest } \\
\text { radiography }\end{array}$ & 0.1 \\
\hline 99mTc-radionuclide cystography & 0.1 \\
\hline Mammography & 0.4 \\
\hline Lumbar spine radiography & 1.5 \\
\hline Head CT & 2.0 \\
\hline 99mTc-MAG3 renal scanning & 2.7 \\
\hline Intravenous urography & 3.0 \\
\hline 99mTc-MDP bone scanning & 4.2 \\
\hline 123I-metaiodobenzylguanidine scanning & 4.8 \\
\hline 99mTc-ethylcysteinate dimer brain scanning & 5.7 \\
\hline Pelvic CT & 6.0 \\
99mTc-sestamibi for stress/rest cardiac & 6.7 \\
\hline scanning & \\
Chest CT & 7.0 \\
\hline Coronary angiography & 7.0 \\
\hline 18F-FDG PET scanning & 7.4 \\
\hline Abdominal CT & 8.0 \\
\hline Coronary angioplasty with stent placement & 15.0 \\
\hline
\end{tabular}

Radiopharmaceutical doses are from Table 1 except ${ }^{99 m}$ Tcradionuclide cystogram dose (24-27). Radiographic doses are from Mettler et al. (23).

Table 1, except for ${ }^{99 \mathrm{~m}}$ Tc-radionuclide cystography, which is performed only in children (27).

\section{Dosimetry of CT}

Hybrid imaging, including PET/CT and SPECT/CT, has become a standard component of medical imaging $(28,29)$. The combination of the anatomic information from CT and the functional information from PET and SPECT provides clinicians with essential information not attainable from either study alone. In addition, the CT information can be used for attenuation correction and anatomic localization.

In CT, x-rays are emitted that expose the patient to ionizing radiation. The dosimetry associated with CT in PET/CT has been described previously (30-32). The number of x-rays emitted can be controlled by adjusting various $\mathrm{CT}$ acquisition parameters, including the tube voltage $(\mathrm{kVp})$ and the tube current-time product (mAs). The tube current may be modulated during acquisition such that fewer x-rays are emitted through thinner or less attenuating parts (e.g., the lungs) of the body. CT may be acquired over a limited field of view. For example, a SPECT/CT scan looking for parathyroid adenomas may include the neck and thorax, whereas an oncologic PET/CT scan may extend from the base of the patient's skull to the mid thighs. When CT is acquired in a helical fashion, the speed of the traversing bed defines the time required to scan the selected volume. This is expressed as pitch, which is the distance traversed by the bed during 1 rotation of the x-ray tube divided by the col- limated beam width. Thus, a higher pitch (faster bed speed) leads to a lower dose.

The radiation dose delivered by $\mathrm{CT}$ to defined locations within standard cylindric acrylic phantoms (16- and 32-cm diameter for the head and whole-body phantoms, respectively) is referred to as the CT dose index (CTDI in units of mGy). If CTDI is averaged over several locations within the phantom (central and peripheral) and normalized by the pitch, it is referred to as $\mathrm{CTDI}_{\mathrm{vol}}$. The dose-length product (in units of $m G y-c m$ ) is the product of the $\mathrm{CTDI}_{\mathrm{vol}}$ and the axial length of the CT acquisition. Values of $\mathrm{CTDI}_{\mathrm{vol}}$ and dose-length product are typically displayed on the CT operator's console during an acquisition.

These values do not represent the radiation dose to a particular patient but to the standard phantoms. A series of anthropomorphic phantoms composed of tissue-equivalent material has been used to estimate the radiation dose from CT in both PET/CT and SPECT/CT to patients of varying sizes (Table 3) (30). For the same CT acquisition parameters, the dose to a newborn is approximately twice that to a medium-sized adult. Several groups have developed and used computerized phantoms for the estimation of CT dose to children and have corroborated these findings $(17,18)$. Therefore, CT acquisition parameters should be reduced for smaller patients $(33,34)$. As with radiopharmaceutical dosimetry, these estimates are averages for patients of different ages, and the radiation dose to a particular individual may vary.

\section{RADIATION RISK IN CHILDREN}

Assessing the risk associated with exposure to ionizing radiation involves applying models that use both epidemiologic and biologic data to extrapolate from the available data to the dosimetric region of interest. Such extrapolation is not straightforward. Much of the current understanding about the risk of exposure to ionizing radiation for humans is based on the Life Span Study of the survivors of the bombings of Hiroshima and Nagasaki as reported by the Radiation Effects Research Foundation (35-39). A recent review of these data (through 1997) showed that the cancers

\section{TABLE 3}

Estimate of Radiation Dose to Anthropomorphic Phantom from CT Component of Hybrid Imaging as Function of Patient Size and Tube Current

\begin{tabular}{lccc}
\hline Patient size & $40 \mathrm{~mA}$ & $80 \mathrm{~mA}$ & $160 \mathrm{~mA}$ \\
\hline Newborn & 5.05 & 10.1 & 20.20 \\
1-y-old & 4.45 & 8.89 & 17.78 \\
5-y-old & 4.08 & 8.16 & 16.31 \\
10-y-old & 3.67 & 7.35 & 14.69 \\
Medium adult & 2.55 & 5.10 & 10.19
\end{tabular}

All data were acquired with tube voltage of $120 \mathrm{kVp}$, rotation speed of $0.8 \mathrm{~s}$, and pitch of 1.5:1. All data were acquired with $160 \mathrm{~mA}$ and linearly scaled for the various tube currents shown here (30). 
occurring in excess included lung, breast, thyroid, bone, and leukemia. Between 1950 and 1997, 87,000 people were followed, and it is estimated that there have been 440 excess cases of cancer in this population due to radiation exposure, with a clear relationship between cancer risk and the amount of radiation received (Fig. 3) (36). This study is often characterized as a study of high dose, because the subjects who received higher doses $(>0.2 \mathrm{~Gy})$ represented most of the cases of excess cancer. However, $80 \%$ of the subjects received less than $0.1 \mathrm{~Gy}$. Ten percent of the total excess cancer deaths occurred in the population that received $0.005-0.10 \mathrm{~Gy}$ (an excess of 44 of a total of 3,277 cancer deaths in a population of 32,000 subjects), a dose range that is similar to that received by many of our patients, particularly those receiving more than 1 scan.

The Life Span Study also demonstrates that the risk of ionizing radiation varies with both age and sex. Younger subjects had a significantly higher risk than older subjects. This higher risk has been attributed to 2 causes: first, the tissues of younger subjects are more radiosensitive because they are actively growing and, second, younger subjects have a longer life span than adults, allowing a longer time for the risk to be realized. Girls demonstrated a higher risk for cancer induction than boys by almost $50 \%$, which is, in large part, attributable to the excess risk of breast cancer in this population. These higher sensitivities in younger subjects, and girls specifically, will be discussed in the context of evaluating the radiation risk in pediatric nuclear medicine.

Many other epidemiologic studies have assessed risk from ionizing radiation. These include studies of occupational exposure in radium dial painters, uranium and hard rock miners, and radiation shipyard workers. Studies of medical exposure have included patients receiving radiation as treatment for a variety of conditions, including tinea capitis, ankylosing spondylitis, and thymus enlargement,

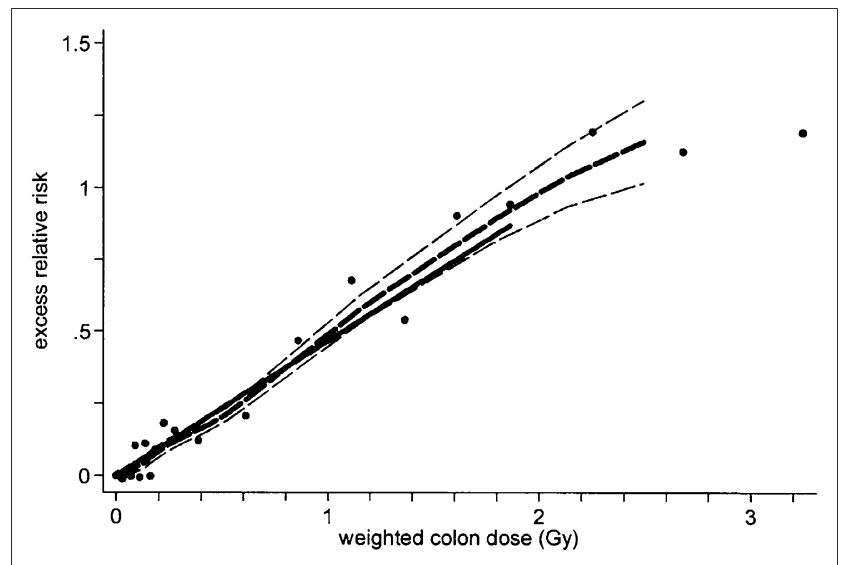

FIGURE 3. Dose response for incidence of solid cancer as function of weighted colon dose as derived from Life Span Study. Weighted colon dose was used as surrogate for whole-body dose within Life Span Study. Solid line demonstrates linear fit through data. (Reprinted with permission of (36).) and patients undergoing multiple fluoroscopic sessions during treatment for tuberculosis $(3,5)$. In general, the findings from these studies have tended to corroborate the findings from the Life Span Study. For example, several epidemiologic studies, including the Life Span Study, are in reasonable agreement about the estimated radiation risk for thyroid cancer (Fig. 4). Most of these exposures were in children (40).

Epidemiologic approaches are limited in their ability to demonstrate small health effects, even if these effects have a substantial impact on the health of the population. For example, it is difficult for epidemiology to demonstrate that a particular exposure led to an increased disease rate of a few percentage points. To have adequate statistical power, such as study would need to involve a large number of subjects (hundreds of thousands or perhaps millions). However, in such a large study it becomes exceedingly difficult to control the heterogeneity of the population, making the study all the more difficult. The Life Span Study has followed subjects of both sexes and all ages who had a wide range of exposure levels. This study has provided a wealth of information, but its design has made it difficult to address specific, focused questions about radiation risk, particularly at low doses.

For these reasons, epidemiologic findings should be augmented by biologic investigations that use experimental animals or cell cultures, allowing greater control over experimental conditions. However, extrapolating from the results of these experiments to the human population can present many challenges. For example, if the results of biologic experiments are contradictory, it can be difficult to draw a useful inference in the context of risk to human populations. Despite this, biologic experiments have pro-

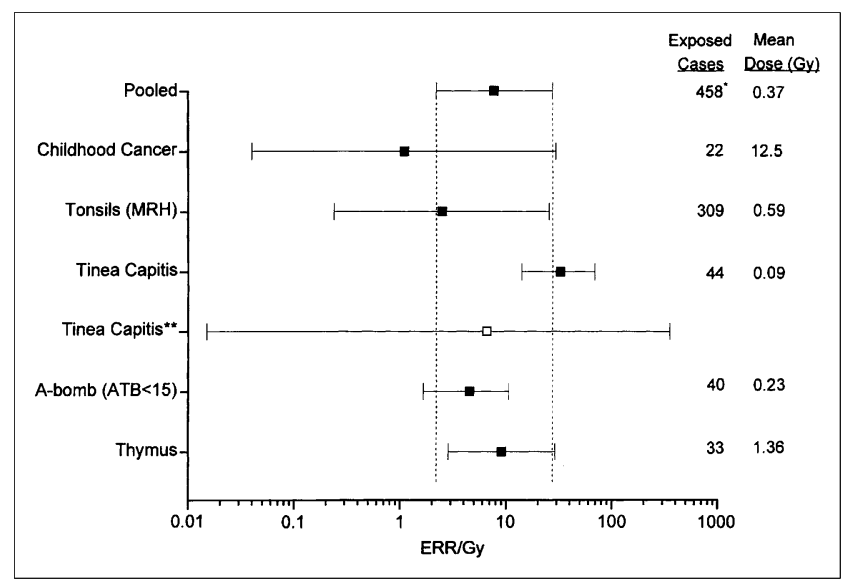

FIGURE 4. Excess relative risk per dose (ERR/Gy) of thyroid cancer from 6 epidemiologic studies including Life Span Study. Excess relative risk is traditional relative risk minus 1 (e.g., if relative risk is 2.5 , then excess relative risk is 1.5 ) and represents fractional increase in natural disease rate as result of exposure in question. $\square=$ value adjusted for nonzero intercept because lower bound was less than zero. Most subjects in these studies were children. Results show consistency. (Reprinted with permission of (40).) 
vided much insight into the nature of the biologic effects of ionizing radiation.

The target of greatest interest with respect to the health effects of ionizing radiation is the DNA within the cell (41). This damage can be direct when ionizations caused by the radiation lead to specific changes within the DNA. However, in most cases of interest to nuclear medicine, the damage is indirect: the passage of the radiation leads to radiolysis of water molecules within the tissue, causing the formation of free radicals that subsequently interact with the DNA. In some cases, the damaged component of the genetic material is essential for cell survival, and the cell may die or not be able to undergo proper mitosis. The removal of these cells will not contribute to late radiation effects such as carcinogenesis. Instead, late effects occur when the cell survives the initial genetic damage. The consequences of this damage manifest later, perhaps decades after the initial exposure. Such late effects may result from genomic instability due to the initial radiation damage. Cells that are growing rapidly and undergoing mitosis at a higher rate may be more susceptible to late radiation effects than those that are growing more slowly.

There also may be secondary effects of radiation exposure that alter the level of cellular damage within exposed tissue. Some cellular experiments have demonstrated a bystander effect in which cells that have been specifically damaged by the radiation can lead to deleterious alterations in neighboring cells. In this case, the tissue damage would be higher than would be expected from considering only the particular cells damaged by sparsely ionizing radiation. On the other hand, there may be situations in which a low radiation exposure leads to a lessened response.

\section{EVALUATION OF RADIATION RISK FOR PEDIATRIC NUCLEAR MEDICINE}

In 2007, the Biologic Effects of Ionizing Radiation Committee of the National Academy of Sciences of the United States reviewed the current epidemiologic and biologic data and developed models of radiation risk as a function of dose, sex, and age at the time of exposure as reported in the biological effects of ionizing radiation (BEIR) VII phase 2 report (3). As seen in Figure 3, to make inferences with respect to risk in the dose realm of interest for nuclear medicine, one must extrapolate from the more defined data at high dose levels (42). Figure 5 illustrates several extrapolation models, including a simple linear model $(\mathrm{R} \propto \mathrm{D}$, where $\mathrm{R}$ is risk and $\mathrm{D}$ is dose), a super linear model, and a hormetic model. There may also be a threshold dose below which there is no risk, presumably because of repair of radiation damage. A linear quadratic model $\left(\mathrm{R} \propto \mathrm{aD}+\mathrm{bD}^{2}\right.$ ) (not shown) may also be considered. On the basis of the available data, the BEIR VII phase 2 report recommended the use of a linear no-threshold model for solid tumors and a linear quadratic model for leukemia. The choice of model is a subject of some controversy (4346). The Academie de Sciences-Institut de France, review-

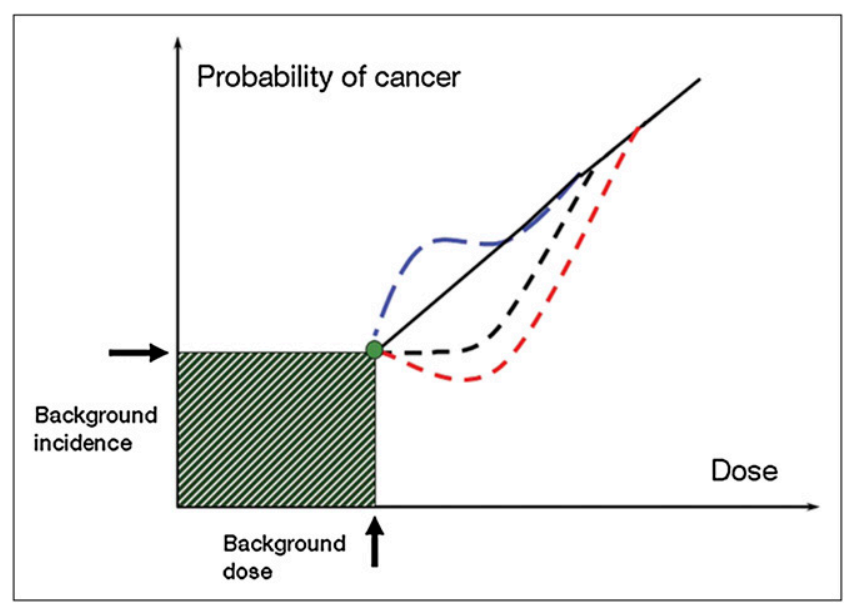

FIGURE 5. Extrapolation models for estimating radiation risk at low dose. Image shows 4 models: linear, no threshold (solid black line); linear, with threshold (dashed black line); super linear (blue line); and hormetic (red line) (42).

ing the same material, reached a very different conclusion: "While [the linear no-threshold model] may be useful for the administrative organization of radioprotection, its use for assessing carcinogenic risks, induced by low doses, such as those delivered by diagnostic radiology or the nuclear industry, is not based on valid scientific data." (47)

Despite these different viewpoints, it is considered prudent to use the models recommended by the BEIR VII phase 2 report (3). The risk estimates provided by these models can be presented in terms of either absolute or relative risk. With absolute risk, the number of excess cases over the natural risk is estimated, whereas with relative risk the percentage increase in the natural risk is calculated. Figure 6 plots the lifetime excess absolute risk of mortality from cancer resulting from a whole-body exposure of $10 \mathrm{mSv}$ as a function of age at exposure for both males and females. The value of $10 \mathrm{mSv}$ was chosen because it is slightly higher than the effective dose estimation for many pediatric nuclear medicine procedures. Table 4 lists the lifetime excess absolute risk of mortality for breast, lung, and colon cancer; total solid tumors; and leukemia for a newborn, 10-y-old, and 40-y-old from a whole-body exposure of $10 \mathrm{mSv}$. For solid tumors, there is a higher risk associated with sex, as well as for those exposed at a younger age. For a fixed radiation dose, a 10-y-old experiences about twice the risk and a newborn about 3 times the risk of a 40-y-old. In addition, the risk is approximately 50\% higher for girls than for boys. Conversely, the risk of mortality from leukemia is higher for males as compared with females and does not vary with age. Combining these factors, the BEIR VII models indicate that the sex-averaged lifetime risk of dying of cancer from exposure to $10 \mathrm{mSv}$ ( $1 \mathrm{rem})$ is approximately 1 in 700, 1,000, and 2,000 for a 1-y-old, 10-y-old, and 40-y-old, respectively (Fig. 6).

Several recent reports have related radiation risk to medical imaging, typically CT but in some cases nuclear 


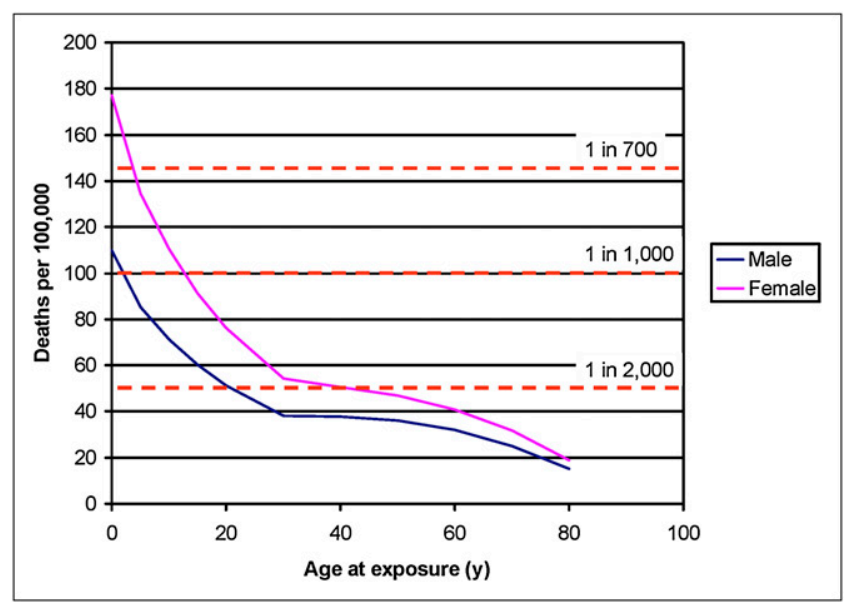

FIGURE 6. Lifetime attributable risk of cancer death as function of age at time of exposure and sex resulting from 10-mSv exposure. Dashed lines indicate attributable risk levels of 1 in 700,1 in 1,000, and 1 in 2,000 corresponding to sex-averaged risk for 1-, 10-, and 40-y-old, respectively (3).

medicine (48-57). Some reports specifically addressed exposure of pediatric patients $(58-60)$. The risk to patients of a certain age for a particular radiologic or nuclear medicine procedure will depend on the dose delivered to each radiosensitive organ and the risk of cancer induction for that organ. When the administered activity is scaled by weight, the radiation dose to the patient is slightly less for smaller patients than for larger patients (Table 1). Conversely, the radiation risk per unit dose increases in younger patients. To some extent, these 2 effects compensate for each other. Table 5 uses as an example a renal ${ }^{99 \mathrm{~m} T c-M A G 3}$ scan. Patients of several ages are considered, and the administered activity based on scaling by weight is given, from which the effective dose is estimated. From Figure 6, the radiation risk for cancer mortality is estimated and multiplied by the effective dose to provide an estimate of the risk for that age patient. This is a rough estimate, and a more careful analysis would have used organ doses rather than the effective dose. This example suggests that a convenient working model may be that children experience a similar risk that is roughly twice the risk of an adult of developing cancer from nuclear medicine procedures.

\section{COMMUNICATION OF RISK TO PARENTS AND CHILDREN}

Media reports about the medical use of radiation have increased, and the general public has shown increased interest in this topic. It is more important than ever that nuclear medicine practitioners-including physicians, physicists, technologists, and other members of patient care teams-be able to effectively communicate with their physician colleagues and with patients and families about the medical use of radiation, the level of radiation exposure, and its potential risk. Perceptions about radiation can vary widely among scientists and the public (61). The awareness
TABLE 4

Lifetime Excess Attributable Risk of Mortality per 100,000 Individuals for 10-mSv Whole-Body Exposure (3)

\begin{tabular}{lcccc}
\hline Tumor type & Sex & Newborn & 10-y-old & 40-y-old \\
\hline Breast & F & 27.4 & 16.7 & 3.5 \\
Lung & F & 64.3 & 44.2 & 21.2 \\
& M & 31.8 & 21.9 & 10.7 \\
Colon & F & 10.2 & 7.3 & 3.7 \\
& M & 16.3 & 11.7 & 6.0 \\
All solid & F & 172 & 105 & 45.5 \\
& M & 103 & 64.1 & 31.0 \\
\hline Leukemia & $\mathrm{F}$ & 5.3 & 5.3 & 5.2 \\
& $\mathrm{M}$ & 7.1 & 7.1 & 6.7 \\
\hline
\end{tabular}

of radiation protection among our pediatrician colleagues is generally low, and thus we need to be prepared to discuss these issues and to answer any questions they may have (62).

When patients or their parents ask about radiation dose, what they are really asking about is risk. Often, it is no longer sufficient to merely indicate that our procedures are safe. We need to reassure our patients and their families that we understand their concerns and that every precaution has been taken to ensure that the appropriate test is being performed, one that will provide their doctor with the best diagnostic information and will expose the patient to the least radiation possible. Experience has shown that parents can be provided with dosimetric and risk information, including a discussion that exposure to radiation may possibly lead to an increased risk of cancer, and that such information typically does not adversely affect their willingness to have their child undergo appropriate medical imaging tests $(63,64)$.

Many methods can be used in explaining these concepts. The "Image Gently" campaign was developed by the Alliance for Radiation Safety in Pediatric Imaging, which includes the Society for Pediatric Radiology, the American College of Radiology, the American Association of Physicists in Medicine, the Society of Nuclear Medicine (SNM), and the SNM Technologist Section. It initially focused on CT exposure but has now broadened its attention to include other modalities, such as nuclear medicine. This campaign seeks to increase awareness about lowering radiation dose

TABLE 5

Administered Activity and Estimates of Radiation Dose and Additional Relative Risk for ${ }^{99 m}$ Tc-MAG3

\begin{tabular}{ccccc}
\hline $\begin{array}{c}\text { Age } \\
(\mathrm{y})\end{array}$ & $\begin{array}{c}\text { Weight } \\
(\mathrm{kg})\end{array}$ & $\begin{array}{c}\text { Administered activity } \\
(\mathrm{MBq})\end{array}$ & $\begin{array}{c}\text { Effective dose } \\
(\mathrm{mSv})\end{array}$ & $\begin{array}{c}\text { Risk } \\
(\%)\end{array}$ \\
\hline 1 & 9.7 & 53.8 & 1.2 & 0.04 \\
5 & 19.8 & 109.9 & 1.3 & 0.03 \\
\hline 10 & 33.2 & 184.3 & 2.2 & 0.05 \\
15 & 56.8 & 315.2 & 2.8 & 0.05 \\
20 & 70.0 & 388.5 & 2.7 & 0.04 \\
40 & 70.0 & 388.5 & 2.7 & 0.02 \\
\hline
\end{tabular}


in the imaging of children and to provide information for parents and patients about medical imaging and radiation risk (65,66). A group representing the SNM, the Society for Pediatric Radiology, and the American College of Radiology in conjunction with Image Gently developed a brochure for patients and families that discusses the value of nuclear medicine, the hazards of radiation exposure, and efforts to reduce dose (67). Some nuclear medicine clinics may want to develop their own materials that address the issues and concerns of their unique patient population. For example, clinics with a significant pediatric oncologic practice may want to include in their patient education materials a specific discussion about PET. Before a nuclear medicine procedure, many patients and their families may ask specific questions about the radiation involved and what effect it might have on their health. Nuclear medicine professionals should be prepared to answer these questions in a clear and assuring manner. It is best if all members of the nuclear medicine clinic have discussed these issues ahead of time so that the patient receives consistent explanations. It should be clear which questions the technologists are comfortable answering themselves and when the discussion should be referred to a physician, a medical physicist, or an expert in radiation safety. Thus, the patient can be presented with clear, concise, and correct information in a confident manner.

The most effective way to communicate issues about radiation risk to the public continues to be a topic of discussion (68). It is likely that many parents have seen news or Internet reports about radiation exposure from medical imaging. Thus, parents and older children come equipped with greater knowledge about radiation and imaging procedures than in the past. Younger children may not have the conceptual ability to comprehend these issues, but teenage patients may very well have seen media coverage of these subjects or even have discussed them in the classroom. They may have some basic understanding and know that exposure to radiation may cause harm, but they will want to know how this specifically applies to their situation. A reasonable approach is to discuss with patients and their families that we will be administering a small amount radioactivity to perform a study that emits radiation similar to that emitted by $\mathrm{x}$-ray machines. This exposure might lead to a slight increase in the risk of contracting cancer sometime in their lifetime. Quantitative estimates of the effective dose, in millisieverts, associated with a procedure may be of limited use, as most patients or parents are unlikely to have a context in which to interpret this information. Comparing the effective dose of a nuclear medicine study with other radiologic procedures (such as a chest radiograph or a CT scan) may also be of little help because the patients or parents may not know if these studies represent small or large exposures. Probably more easily understood would be an explanation that the radiation dose from the procedure is generally in the range of many other radiologic tests and is on the same order as that individuals get from natural background radiation in $1 \mathrm{y}$. In addition, the dose to the parent from the radioactivity administered to his or her child is on the order of the radiation one would receive during a transcontinental flight (4). Consistent with the linear no-threshold model recommended by BEIR VII, one should explain that each exposure carries its own small risk and that there is no cumulative threshold above which the patient is considered to be at greater, significant risk.

Sometimes, patients and their families may request a more quantitative estimate of the risk, and one needs to consider how best to communicate this. As an example, consider the risk of a 10-y-old who receives a 99mTc-methylene diphosphonate (MDP) bone scan with an estimated effective dose of $3.9 \mathrm{mSv}$ (Table 1). The probability of potentially developing fatal cancer as a result of this exposure has been estimated to be about 1 in 2,560. One study suggested that describing the magnitude of risk in pictorial terms such as a pie chart may be the most effective approach for children and parents (68). A pictorial approach is demonstrated in Figure 7, which consists of 2,500 small circles each representing a child who received a ${ }^{99 \mathrm{~m}} \mathrm{Tc}-\mathrm{MDP}$ bone scan. The red star in the lower right corner represents the 1 individual in the 2,500 who may develop fatal cancer later in life. Using a verbal descriptive approach (e.g., if 2,500 children receive a ${ }^{99 \mathrm{~m}}$ Tc-MDP bone scan, 1 of those may develop fatal cancer as a result) or representing the risks in terms of percentages (e.g., $0.04 \%$ of children receiving a ${ }^{99 \mathrm{~m}} \mathrm{Tc}-\mathrm{MDP}$ bone scan may develop fatal cancer) also was found to be effective; however, subjects were, in general, less confident that they

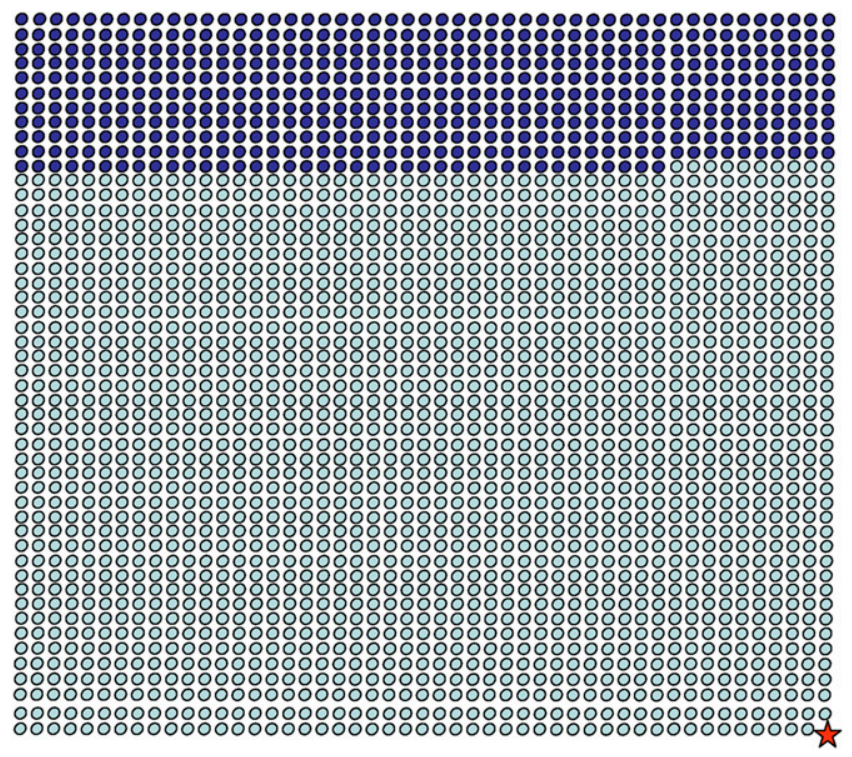

FIGURE 7. Demonstration of 1 in 2,500 risk in comparison to 550 in 2,500. For example of 10 -y-old receiving $99 \mathrm{mT}$ T-MDP bone scan, excess attributable risk for cancer death is 1 in 2,500 . In this figure, there are 2,500 small circles. Lone red star at lower right represents 1 case in 2,500 in which bone scan patient may contract fatal cancer. In addition, there are 550 dark blue circles that represent number of the original 2,500 that will naturally die of cancer $(22 \%)$. 
understood the information being provided than with graphic approaches. Least effective was providing the data as a proportion (e.g., 1 in 2,500 children receiving a 99mTc-MDP bone scan may contract fatal cancer); many children and parents mistakenly interpret 1 in 500 as a higher risk than 1 in 100. Presenting risk information in a consistent fashion was found to be more effective than presenting a mixed approach.

Patients and parents may need some insight as to the context in which to view this risk information. One can state that practically all medical procedures and interventions, even the administration of prescription medicines, have side effects and risks associated with them, and radiation exposure from medical imaging is no different. Within the spectrum of medical interventions, nuclear medicine is considered a safe procedure. One can also compare the cancer risk associated with radiation exposure to the natural risk of cancer. In the United States, approximately $22 \%$ of the population will die of cancer (69). This risk can be compared with the $0.04 \%$ increased risk for a child receiving a ${ }^{99 \mathrm{~m}} \mathrm{Tc}-\mathrm{MDP}$ bone scan. Another way of presenting these data is that of 2,500 children who receive a ${ }^{99 m}$ Tc-MDP bone scan, approximately 550 will naturally die of cancer sometime in their life and 1 additional case may be due to the radiation exposure from the ${ }^{99 \mathrm{~m}} \mathrm{Tc}-\mathrm{MDP}$ bone scan. This is illustrated in Figure 7, where the 550 dark blue circles represent the individuals who will naturally die of cancer in their lives.

An alternative approach would be to compare the risk associated with radiation from medical imaging to other lifetime risks. Table 6 lists the lifetime risk of death from several activities over the entire U.S. population (69). In the table, the value 304 for the lifetime risk of dying while riding in a car indicates that 1 of 304 Americans will die as a result of an accident while riding in a car during his or her lifetime. Also listed are estimates of the risk of cancer

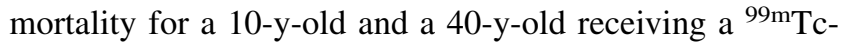
MDP bone scan and a ${ }^{18} \mathrm{~F}$ FDG PET scan. In this context, the additional risk for cancer fatality for a 10-y-old (1 in 1,500 from a PET scan and 1 in 2,500 from a bone scan) compares to other uncommon causes of death such as being caught in a fire or falling down a flight of stairs and is much less likely than dying as the result of an assault or an accident while riding in a car or walking. Thus, it can be shown that exposure to radiation from nuclear medicine presents a very low risk to the patient.

The best way to discuss radiation risk depends on the individual circumstances. For example, an individual who recently lost a family member to a fall from a ladder or bicycle accident may overestimate the likelihood of these rare events. However, it is essential to provide accurate information in a clear, concise, and professional manner. Any discussion of risk should include the specific benefits of the procedure to the patient. Patients and families want to be assured that the benefits far outweigh any health risk of the procedure. For each procedure, the nuclear medicine
TABLE 6

Lifetime Risk of Death from Everyday Activities in United States (69)

\begin{tabular}{lr}
\hline \multicolumn{1}{c}{ Activity } & Lifetime risk \\
\hline Assault & 214 \\
\hline Accident while riding in car & 304 \\
\hline Accident as pedestrian & 652 \\
\hline Choking & 894 \\
\hline Accidental poisoning & 1,030 \\
\hline Drowning & 1,127 \\
Exposure to fire or smoke & 1,181 \\
\hline Cancer from ${ }^{18}$ F-FDG PET scan (10-y-old) & 1,515 \\
\hline Falling down stairs & 2,024 \\
Cancer from ${ }^{99 m T c-M D P ~ b o n e ~ s c a n ~(10-y-o l d) ~}$ & 2,560 \\
\hline Cancer from ${ }^{18}$ F-FDG PET scan (40-y-old) & 2,700 \\
\hline All forces of nature & 3,190 \\
Accident while riding bike & 4,734 \\
Cancer from ${ }^{99 m T c-M D P ~ b o n e ~ s c a n ~(40-y-o l d) ~}$ & 4,760 \\
Accidental firearms discharge & 6,333 \\
Accident while riding in plane & 7,058 \\
Falling off ladder or scaffolding & 10,606 \\
\hline Hit by lightning & 84,388 \\
\hline
\end{tabular}

Lifetime risk of 304 for accident while riding in car indicates that 1 of every 304 Americans will die as result of accident while riding in car during his or her lifetime.

staff should be clear in describing the importance of the specific information provided by the study.

\section{DOSE REDUCTION IN PEDIATRIC NUCLEAR MEDICINE}

Pediatric nuclear medicine should be optimized to provide the necessary clinical information while reducing the radiation risk to the patient $(70,71)$. A study should be performed only if that study is appropriate for answering the clinical question being asked. The radiation dose for each procedure should be kept as low as possible. It is clear from the MIRD equation (Eq. 1) that the most easily controlled parameter affecting radiation dose from nuclear medicine is the administered activity. Sometimes, clearance of the radioactivity can be slightly modified by keeping patients well hydrated and having them empty their bladders as often as possible. Unfortunately, the administered activity in pediatric patients has not been standardized. A survey was conducted among North American pediatric institutions inquiring as to the maximum activity administered to larger patients (e.g., $70 \mathrm{~kg}$ ) for 16 commonly performed procedures, their methods of determining the activity for smaller patients, and their minimum activity administered to very small patients below which they would consider the study inadequate irrespective of patient size (72). Most centers determined their activity for smaller patients on the basis of body weight. However, there was a large variation in the dosing schemes across all protocols among the institutions. The maximum administered activity and the activity per body mass varied by a factor of 3 on average and as much as a factor of 10 across the 16 protocols. The minimum administered 
activity varied by a factor of 10 on average and as much as a factor of 20 for 1 protocol.

The radiopharmaceutical dosing schedule at many institutions was established several years ago on the basis of experience, the patient population, and the imaging equipment available at the time. The assumptions may no longer be valid given changes in practice and advances in instrumentation. For example, many institutions no longer evaluate the perfusion phase of the ${ }^{99 \mathrm{~m}} \mathrm{Tc}-\mathrm{MAG} 3$ renogram. Without the necessity to have adequate counts for the perfusion-phase images, the administered activity can be reduced without compromising the analysis and interpretation of the study.

Advances in instrumentation also may facilitate reducing the administered activity. For example, with dual-detector rather than single-detector SPECT systems, the administered activity can be reduced. Focused collimators developed for use with cardiac SPECT may be applicable to imaging smaller patients as well. In PET, 3-dimensional (3D) rather than 2-dimensional acquisition leads to increased sensitivity. Although 3D PET is more susceptible to scatter and random coincidences from activity outside the field of view, these are less of an issue with small patients.

Improvements in reconstruction algorithms and image reconstruction allow for adequate image quality with significantly fewer counts, allowing the study to be performed with less administered activity. Ordered-subset expectation maximization iterative reconstruction with $3 \mathrm{D}$ resolution recovery has been investigated with respect to its use with pediatric ${ }^{99 \mathrm{~m}} \mathrm{Tc}-\mathrm{MDP}$ bone scans and ${ }^{99 \mathrm{~m}} \mathrm{Tc}$-dimercaptosuccinic acid renal scans $(73,74)$. In both cases, adequate image quality was achieved with half the counts (and thus half the administered activity) using 3D ordered-subset expectation maximization as compared with conventional filtered backprojection. When adaptive filtering for noise reduction was applied to ${ }^{99 \mathrm{~m} T c-M A G 3}$ renal studies, the administered activity could be reduced by as much as $80 \%$ (75).

With hybrid imaging, the radiation dose from CT also needs to be considered $(32,76)$. Chawla et al. reviewed the cumulative dose received from ${ }^{18} \mathrm{~F}-\mathrm{FDG}$ PET/CT by children (aged 1.3-18 y) being treated for cancer from 2002 to 2007 (77). In this study, the CT component was acquired with diagnostic quality. The average dose per PET/CT scan was estimated to be $24.8 \mathrm{mSv}$. In their population, the average number of PET/CT scans per patient was 3.2 (range, 1-14 scans), for an average cumulative dose of $78 \mathrm{mSv}$ (range, 6.2-399 $\mathrm{mSv}$ ). The CT in this study was acquired as a diagnostic study, and it is possible to substantially reduce the radiation dose. Alessio et al. developed a scheme for pediatric ${ }^{18} \mathrm{~F}$-FDG PET/CT in which a diagnostic CT scan was previously acquired and CT was used for attenuation correction and anatomic correlation (78). Using the Broslow-Luten color scheme to scale the CT tube current (tube voltage constant at $120 \mathrm{kVp}$ ) and scaling the ${ }^{18} \mathrm{~F}-\mathrm{FDG}$ administered activity by patient weight, the effective dose from the ${ }^{18} \mathrm{~F}-\mathrm{FDG}$ PET/CT scan was in the range of $8-13 \mathrm{mSv}$. One may also consider limiting the PET/CT scan to the portion of the body that is of interest. The dose from ${ }^{18} \mathrm{~F}$-FDG will not be reduced, but the dose from the CT will be affected. Very low-dose CT (e.g., $80 \mathrm{kVp}$ and $5 \mathrm{mAs}$; perhaps a 70 times lower dose than for diagnostic CT) can be used for attenuation correction (30). Therefore, one could scan the portion of the body that is of greatest interest using diagnostic CT and scan the remainder of the body with low-dose parameters. If there is a suggestive finding on the PET scan, the additional area could then be CT-scanned at diagnostic levels.

There have been several efforts to reduce the dose in pediatric nuclear medicine. For PET, Accorsi et al. estimated the optimum administered activity on the basis of the noise-equivalent counting rate (79). The European Association of Nuclear Medicine has developed a pediatric scheme that uses a nonlinear approach to scaling the administered activity as a function of patient size (80). More recently, a group of experts representing the SNM, Society for Pediatric Radiology, and American College of Radiology working with the Image Gently program reached a consensus on a scheme that scaled the administered activity by the patient's weight (81). Any of these can be used to develop a dosing scheme that is most appropriate for each particular pediatric nuclear medicine clinic.

\section{SUMMARY}

Pediatric nuclear medicine provides invaluable diagnostic information for many clinical specialties. During these imaging procedures, patients are exposed to ionizing radiation and the small risk associated with its use. Recently, reports on the medical use of radiation have led to increased interest by the general public. There is particular concern for young children because they are more sensitive to radiation than adults. For these reasons, practitioners of pediatric nuclear medicine need to have a basic understanding of radiation risk and dosimetry, particularly of how they apply to nuclear medicine procedures, and of how best to communicate this information to patients and their families. We need to assure our patients that we understand these issues and are committed to obtaining excellent clinical results using the lowest possible administered activities and, thus, the minimum necessary risk.

\section{REFERENCES}

1. Treves ST. Pediatric Nuclear Medicine. New York, NY: Springer; 2007.

2. Treves ST, Baker A, Fahey FH, et al. Nuclear medicine in the first year of life. J Nucl Med. 2011;52:905-925.

3. Committee to Assess Health Risks from Exposure to Low Levels of Ionizing Radiation, National Research Council. Health Risks from Exposure to Low Levels of Ionizing Radiation: BEIR VII Phase 2. Washington, DC: National Research Council of the National Academies; 2006.

4. National Council on Radiation Protection and Measurement. Ionizing Radiation Exposure of the Population of the United States: Report NCRP 160. Washington, DC: National Council on Radiation Protection and Measurement; 2009.

5. Sources and Effects of Ionizing Radiation: UNSCEAR 2008 Report. Volume I: Sources-Report to the General Assembly Scientific Annexes A, B. New York, NY: United Nations; 2010. 
6. Mettler FA, Bhargavan M, Faulkner K, et al. Radiologic and nuclear medicine studies in the United States and worldwide: frequency, radiation dose, and comparison with other radiation sources-1950-2007. Radiology. 2009;253:520-531.

7. Dorfman AL, Fazel R, Einstein AJ, et al. Use of medical imaging procedures with ionizing radiation in children: a population-based study. Arch Pediatr Adolesc Med. 2011;165:458-464.

8. Fazel R, Krumholz HM, Wang Y, et al. Exposure to low-dose ionizing radiation from medical imaging procedures. N Engl J Med. 2009;361:849-857.

9. Kowalczyk L. Is all that scanning putting us at risk? Boston Globe. September 14, 2009:G6.

10. Bogdanich W. Radiation overdoses point up dangers of CT scans. New York Times. October 16, 2009:A13.

11. Amis ES, Butler PF, Applegate KE, et al. American College of Radiology white paper on radiation dose in medicine. J Am Coll Radiol. 2007;4:272-284.

12. Amis ES, Butler PF. ACR white paper on radiation dose in medicine: three years later. J Am Coll Radiol. 2010;7:865-870.

13. Loevinger R, Budinger TF. MIRD Primer for Absorbed Dose Calculations (Revised Edition) Reston, VA: Society of Nuclear Medicine; 1991.

14. Bolch WE, Eckerman KF, Sgouros G, Thomas SR. MIRD pamphlet 21: a generalized schema for radiopharmaceutical dosimetry-standardization of nomenclature. J Nucl Med. 2009;50:477-484.

15. Cristy M, Eckerman KF. Specific Absorbed Fractions of Energy at Various Ages. Oak Ridge, TN: Oak Ridge National Laboratories; 1987. ORNL/TM-8381.

16. Stabin MG, Siegel JA. Physical models and dose factors for use in internal dose assessment. Health Phys. 2003;85:294-310.

17. Xu G, Eckerman KF, eds. Handbook of Anatomical Models for Radiation Dosimetry. Boca Raton, FL: CRC Press, 2009.

18. Whalen S, Lee C, Williams J, Bolch WE. Anthropomorphic approaches and their uncertainties to assigning computational phantoms to individual patients in pediatric dosimetry studies. Phys Med Biol. 2008;53:453-471.

19. Nosske D, Blanchardon E, Bolch WE, et al. New developments in internal dosimetry models. Radiat Prot Dosimetry. 2011;144:314-320.

20. Recommendations of the Internal Commission of Radiation Protection: ICRP Publication 26. New York, NY: Pergamon Press; 1977.

21. The 2007 Recommendations of the International Commission on Radiological Protection: ICRP Publication 103. New York, NY: Pergamon Press; 2007.

22. Stabin MG. Internal Dosimetry in Pediatric Nuclear Medicine. 3rd ed. New York, NY: Springer; 2007:513-520.

23. Mettler FA Jr, Huda W, Yoshizumi TT, Mahesh M. Effective doses in radiology and diagnostic nuclear medicine: a catalog. Radiology. 2008;248:254-263.

24. Radiation Dose to Patients from Radiopharmaceuticals: ICRP Publication 53. New York, NY: Pergamon Press; 1988.

25. Radiation Dose to Patients from Radiopharmaceuticals (Addendum to ICRP Publication 53): ICRP Publication 80. New York, NY: Pergamon Press; 1999.

26. Radiation Dose to Patients from Radiopharmaceuticals (A Third Addendum to ICRP Publication 53): ICRP Publication 106. New York, NY: Pergamon Press; 2009.

27. Ward VL, Stauss KJ, Barnewolt CE, et al. Pediatric radiation exposure and effective dose reduction during voiding cystourethrography. Radiology. 2008; 249:1002-1009.

28. Pichler BJ, Judenhofer MS, Wehrl HF. PET/MRI hybrid imaging: devices and initial results. Eur Radiol. 2008;18:1077-1086.

29. Patton JA, Townsend DW, Button BF. Hybrid imaging technology: from dreams and vision to clinical devices. Semin Nucl Med. 2009;39:247-263.

30. Fahey FH, Palmer MR, Strauss KJ, Zimmerman RE, Badawi RD, Treves ST. Dosimetry and adequacy of CT-based attenuation correction for pediatric PET: phantom study. Radiology. 2007;243:96-104.

31. Gelfand MJ, Lemen LC. PET/CT and SPECT/CT dosimetry in children: the challenge to the pediatric imager. Semin Nucl Med. 2007;37:391-398.

32. Fahey FH. Dosimetry of Pediatric PET/CT. J Nucl Med. 2009;50:1483-1491.

33. Donnelly LF, Emery KH, Brody AS, et al. Minimizing radiation dose for pediatric body applications of single-detector helical CT: strategies at a large children's hospital. AJR. 2001;176:303-306.

34. Frush DP. Radiation, CT, and children: the simple answer is. . it's complicated. Radiology. 2009;252:4-6.

35. Preston DL, Shimizu Y, Pierce DA, et al. Studies of mortality of atomic bomb survivors: report 13-solid cancer and noncancer disease mortality: 1950-1997. Radiat Res. 2003;160:381-407

36. Preston DL, Pierce DA, Shimizu Y, et al. Effect of recent changes in atomic bomb survivor dosimetry on cancer mortality risk estimates. Radiat Res. 2004; 162:377-389.

37. Preston DL, Cullings H, Suyama A, et al. Solid cancer incidence in atomic bomb survivors exposed in utero or as young children. J Natl Cancer Inst. 2008;100:428-436.

38. Little MP. Heterogeneity of variation of relative risk by age at exposure in the Japanese atomic bomb survivors. Radiat Environ Biophys. 2009;48:253-262.
39. Walsh L, Kaiser JC. Multi-model inference of adult and childhood leukaemia excess relative risks based on the Japanese A-bomb survivors mortality data (1950-2000). Radiat Environ Biophys. 2011; 50:21-35.

40. Ron E, Lubin JH, Shore RE, et al. Thyroid cancer after exposure to external radiation: a pooled analysis of seven studies. Radiat Res. 1995;141:259277.

41. Hall EJ, Giaccia AJ. Radiobiology for the Radiologist. 6th ed. Philadelphia, PA: Lippincott; 2005.

42. Low Dose and Low Dose-Rate Radiation Effects and Models. Washington, DC: U.S. National Council on Radiation Protection and Measurement; 2008.

43. Friedl AA, Rühm W. LNT: a never-ending story. Radiat Environ Biophys. 2006;44:241-244.

44. Brenner DJ, Sachs RK. Estimating radiation-induced cancer risks at very low doses: rationale for using a linear no-threshold approach. Radiat Environ Biophys. 2006;44:253-256.

45. Land CE. Low-dose extrapolation of radiation health risks: some implications of uncertainty for radiation protection at low doses. Health Phys. 2009;97:407-415.

46. Ulsh BA. Checking the foundation: recent radiobiology and the linear no-threshold theory. Health Phys. 2010;99:747-758.

47. Tubiana M, Aurengo A, Averbeck D, Masse R. Low-dose risk assessment: comments on the summary of the International Workshop. Radiat Res. 2007; 167:742-744.

48. Ron E. Cancer risks from medical radiation. Health Phys. 2003;85:47-59.

49. Brenner DJ, Hall EJ. Computed tomography-an increasing source of radiation exposure. N Engl J Med. 2007;357:2277-2284.

50. Preston RJ. Update on linear non-threshold dose-response model and implications for diagnostic radiology procedures. Health Phys. 2008;95:541-546.

51. Hall EJ, Brenner DJ. Cancer risks from diagnostic radiology. $\mathrm{Br} J$ Radiol. 2008;81:362-378.

52. Berrington de Gonzáles A, Mahesh M, Kim KP, et al. Projected cancer risks from computed tomographic scans performed in the United States in 2007. Arch Intern Med. 2009;169:2071-2077.

53. Smith-Bindman R, Lipson J, Marcus R, et al. Radiation dose associated with common computed tomography examinations and the associated lifetime attributable risk of cancer. Arch Intern Med. 2009;169:2078-2086.

54. Smith-Bindman R. Is computed tomography safe? N Engl J Med. 2010;363:1-4.

55. Brenner DJ, Hricak H. Radiation exposure from medical imaging: time to regulate? JAMA. 2010;304:208-209.

56. Einstein AJ, Weiner SD, Bernheim A. Multiple testing, cumulative radiation dose, and clinical indications in patients undergoing myocardial perfusion imaging. JAMA. 2010;304:2137-2144.

57. Salvatori M, Lucignani G. Radiation exposure, protection and risk from nuclear medicine procedures. Eur J Nucl Med Mol Imaging. 2010;37:1225-1231.

58. Brenner DJ, Ellison CD, Hall EJ, Berdon WE. Estimated risks of radiationinduced fatal cancer from pediatric CT. Am J Roentgenol. 2001;176:289-296.

59. Brody AS, Frush DP, Huda W, et al. Radiation risk to children from computed tomography. Pediatrics. 2007;120:677-682.

60. Robbins E. Radiation risks from imaging studies in children with cancer. Pediatr Blood Cancer. 2008;51:453-457.

61. Jenkins-Smith HC, Silva CL, Murray C. Beliefs about radiation scientists, the public and public policy. Health Phys. 2009;97:519-527.

62. Thomas KE, Parnell-Parmley JE, Haidar S, et al. Assessment of radiation dose awareness among pediatricians. Pediatr Radiol. 2006;36:823-832.

63. Larson DB, Rader SB, Forman HP, Fenton LZ. Informing parents about CT radiation exposure in children: it's OK to tell them. AJR. 2007;189:271-275.

64. Baumann BM, Chen EH, Mills AM. Patient perceptions of computed tomographic imaging and their understanding of radiation risk and exposure. Ann Emerg Med. December 10, 2011 [Epub ahead of print].

65. Goske MJ, Applegate KE, Boylan J. Image Gently(SM): a national education and communication campaign in radiology using the science of social marketing. J Am Coll Radiol. 2008;5:1200-1205.

66. Bulas DI, Goske MJ, Applegate KE, Wood BP. Image Gently: why we should talk to parents about CT in children. AJR. 2009;192:1176-1178.

67. What you should know about pediatric nuclear medicine and radiation safety. Web site of the Alliance for Radiation Safety in Pediatric Imaging. Available at: http://www.pedrad.org/associations/5364/files/Final.IG\%204pgNucMed14.8. 2010.pdf. Accessed May 26, 2011.

68. Ulph F, Townsend E, Glazebrook C. How should risk be communicated to children: a cross-sectional study comparing different formats of probability information. BMC Med Inform Decis Mak. 2009;9:26.

69. Compressed mortality: 1999-2007. CDC WONDER Web site. Available at: http://wonder.cdc.gov/cmf-icd10.html. Accessed May 26, 2011.

70. Hricak H, Brenner DJ, Adelstein SJ, et al. Managing radiation use in medical imaging: a multifaceted challenge. Radiology. 2011; 258:889-905. 
71. Gelfand MJ. Dose reduction in pediatric hybrid and planar imaging. $Q \mathrm{~J} \mathrm{Nucl}$ Med Mol Imaging. 2010;54:379-388.

72. Treves ST, Davis RT, Fahey FH. Administered radiopharmaceutical doses in children: a survey of 13 pediatric hospitals in North America. J Nucl Med. 2008;49:1024-1027.

73. Sheehy N, Tetrault T, Zurakowski D, et al. Pediatric ${ }^{99 \mathrm{~m}}$ Tc-DMSA SPECT using iterative reconstruction with isotropic resolution recovery: improved image quality and reduction in radiopharmaceutical administered activity. Radiology. 2009;251:511-516.

74. Stansfield EC, Sheehy N, Zurakowski D, et al. Pediatric ${ }^{99 \mathrm{~m}} \mathrm{Tc}-\mathrm{MDP}$ bone SPECT with ordered subset expectation maximization iterative reconstruction with isotropic 3D resolution. Radiology. 2010;257:793-801.

75. Hsaio E, Cao X, Zukotynski K, et al. Reduction in radiation dose in MAG3 renography by enhanced planar processing. Radiology. 2011. In press.
76. McCollough $\mathrm{CH}$, Primak AN, Braun N, et al. Strategies for reducing radiation dose in CT. Radiol Clin North Am. 2009;47:27-40.

77. Chawla SC, Federman N, Zhang D, et al. Estimated cumulative radiation dose from PET/CT in children with malignancies: a 5-year retrospective review. $\mathrm{Pe}$ diatr Radiol. 2010;40:681-686.

78. Alessio AM, Kinahan PE, Manchanda V, et al. Weight-based, low-dose pediatric whole-body PET/CT protocols. J Nucl Med. 2009;50:1570-1577.

79. Accorsi R, Karp JS, Surti S. Improved dose regimen in pediatric PET. J Nucl Med. 2010;51:293-300.

80. Lassmann M, Biassoni L, Monsieurs M, et al. The new EANM paediatric dosage card. Eur J Nucl Med Mol Imaging. 2007;34:796-798.

81. Gelfand MJ, Parisi MT, Treves ST. Pediatric radiopharmaceutical administered doses: 2010 North American consensus guidelines. J Nucl Med. 2011;52: $318-322$. 